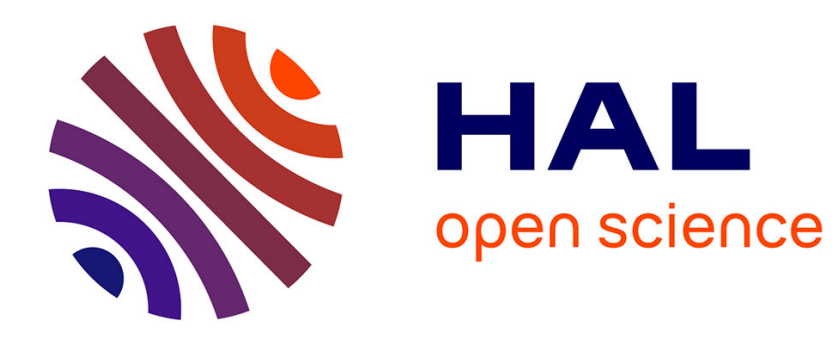

\title{
Conformation changes of a polyelectrolyte chain in a poor solvent
}

\author{
Paul Higgs, Elie Raphael
}

\section{To cite this version:}

Paul Higgs, Elie Raphael. Conformation changes of a polyelectrolyte chain in a poor solvent. Journal de Physique I, 1991, 1 (1), pp.1-7. 10.1051/jp1:1991111 . jpa-00246298

\section{HAL Id: jpa-00246298 https://hal.science/jpa-00246298}

Submitted on 1 Jan 1991

HAL is a multi-disciplinary open access archive for the deposit and dissemination of scientific research documents, whether they are published or not. The documents may come from teaching and research institutions in France or abroad, or from public or private research centers.
L'archive ouverte pluridisciplinaire HAL, est destinée au dépôt et à la diffusion de documents scientifiques de niveau recherche, publiés ou non, émanant des établissements d'enseignement et de recherche français ou étrangers, des laboratoires publics ou privés. 
Classification

Physics Abstracts

36.20

\title{
Short Communication \\ Conformation changes of a polyelectrolyte chain in a poor solvent
}

\author{
Paul G. Higgs $\left({ }^{1}\right)$ and Elie Raphael $\left({ }^{1}, 2\right)$ \\ $\left.{ }^{1}\right)$ Institut Charles Sadron, 6 rue Boussingault, 67083 Strasbourg Cedex, France \\ (2) Laboratoire de Spectrométrie et d'Imagerie Ultrasonores, Université Louis Pasteur, 4 rue Blaise \\ Pascal, 67070 Strasbourg Cedex, France
}

(Received 26 September 1990, accepted 9 November 1990)

\begin{abstract}
Résumé. - Nous étudions le comportement d'une chaîne isolée de polyélectrolyte en mauvais solvant en présence de sel. La conformation de la chaîne résulte de la compétition entre les interactions électrostatiques répulsives et les interactions attractives à courte portée de type volume exclu. Nous montrons en particulier que si le solvant est suffisamment mauvais la chaîne peut passer abruptement d'une forme cylindrique à une forme sphérique pour une certaine concentration critique en sel. Ce changement de conformation n'est pas dú à la condensation des contreions mais provient directement de la compétition entre les interactions s'exerçant entre les monomères.
\end{abstract}

\begin{abstract}
We study theoretically the behaviour of an isolated polyelectrolyte chain in a poor solvent in the presence of salt. The conformation properties of the chain result from the competition between the repulsive Coulomb interactions and the attractive short-range excluded volume interactions. We show in particular that if the solvent is sufficiently poor, the shape of the chain may change abruptly between cylindrical and spherical configurations at a certain critical salt concentration, rather than following a smooth crossover between scaling regimes. The predicted conformational change is not an effect of counterion condensation, and comes only from the competing interactions between the monomers.
\end{abstract}

\section{Introduction.}

Despite much theoretical work on charged polymers [1-9], a general theory of polyelectrolyte solutions fully consistent with experiment has yet to be found. Here we focus on the possible configurations of an isolated polyelectrolyte chain in a poor solvent. The problem is interesting because of the competition between the repulsive long-range Coulomb interactions and the

Current addresses: P.G.H. Service de Physique Théorique, CEN Saclay, 91191 Gif-sur-Yvette Cedex, France.

E.R. Lab. Matière Condensée, Collège de France, 75231 Paris cedex 05, France. 
attractive short-range interactions. The presence of salt reduces the range of the Coulomb interactions and therefore strongly affects the chain conformations. Throughout the paper the term "poor solvent" refers to the quality of the solvent for the equivalent neutral chain. The charged polyelectrolyte may nevertheless be soluble due to the repulsive Coulomb interactions between the charged monomers.

A scaling theory for a polyelectrolyte chain in a $\theta$-solvent in the presence of salt has been given by Khokhlov and Khachaturian (KK) [6]. As the salt concentration increases the overall size of the chain $L$ decreases smoothly, passing through a number of different regimes. A somewhat similar analysis for a polyelectrolyte chain in a salt-free poor solvent (Khokhlov [5]) predicts that $L$ decreases smoothly as the quality of the solvent is decreased. We will see that in the more general case of a polyelectrolyte chain in a poor solvent in the presence of salt, the size $L$ may change abruptly at a certain critical salt concentration.

In this work we consider linear flexible chains, with a large number $(N)$ of monomers per chain and a fraction $f$ of the monomers charged. We assume that the chains are weakly charged $(f \ll 1)$ so that there is no counterion condensation [10], and consider only very dilute solutions, where the chains do not overlap. In this case the alternative type of counterion condensation predicted in reference [5] (avalanche-type counterion-condensation) also does not occur. The number of charges on each chain, and their positions on the chain are moreover assumed to be fixed. The alternative "annealed" case, where charged groups are mobile along the chains, has been introduced in reference [11], and leads to interesting effects. We return to this case in section 4 . We define the Bjerrum length $\ell=\frac{e^{2}}{4 \pi \varepsilon k T}$ and the dimensionless parameter $u=\ell / a$, where $e$ is the electronic charge, $\varepsilon$ the solvent dielectric constant and $a$ the monomer size. In the presence of salt (1-1 electrolyte) of concentration $n$ the Debye screening length $\kappa^{-1}$ is defined by $\kappa^{2}=8 \pi n \ell$.

We recall first the behaviour of a polyelectrolyte chain in a salt free $\theta$-solvent (de Gennes et al [1]). At short length scales the electrostatic interactions are weak and the chain has a Gaussian statistics. It is then convenient to group the monomers into "electrostatic-blobs" of size $D_{0}$ each containing $g_{0}$ monomers $\left(D_{0}=a g_{0}^{1 / 2}\right)$. At larger scales the electrostatic interaction dominates and the chain of blobs is elongated. Balancing the electrostatic energy of a blob by the thermal energy $k T$ one obtains :

$$
g_{0} \sim\left(u f^{2}\right)^{-2 / 3}, \quad D_{0} \sim a\left(u f^{2}\right)^{-1 / 3}
$$

The overall size of the chain of blobs is then :

$$
L_{0} \sim\left(N / g_{0}\right) D_{0} \sim N a\left(u f^{2}\right)^{1 / 3}
$$

Now suppose the chain is in a poor solvent characterized by a negative excluded volume $-\tau a^{3}$ (it is expected that $\tau$ varies linearly with temperature close to the $\theta$ point : $\tau \sim(\theta-T) / \theta$ ). In the absence of charges the attractive short-range interactions would cause the chain to collapse. As was shown by Khokhlov [5], it is still possible to represent the chain as a linear sequence of blobs. At scales shorter than the blob size $D$ the chain has now the statistics of a collapsed neutral chain : $D \sim\left(g / \tau a^{-3}\right)^{1 / 3}$ ( $g$ being the number of monomers per blob). By balancing the electrostatic energy of a blob by the corresponding polymer/solvent interfacial en$\operatorname{ergy}(g e f)^{2} / 4 \pi \varepsilon D \sim\left(k T a^{-2} \tau^{2}\right) D^{2}$ one obtains

$$
g \sim \tau\left(u f^{2}\right)^{-1}
$$


The blob size $D$ remains thus equal to $D_{0}$ but the overall size $L$ of the chain decreases because there are more monomers within each blob :

$$
L \sim N a\left(u f^{2}\right)^{2 / 3} \tau^{-1}
$$

For equation (4) to be valid the blob size must be larger than the thermal correlation length $\xi_{\mathrm{t}}=a \tau^{-1}[14]$ which is the width of the interface between the external solvent and the dense blobs : $\tau>\left(u f^{2}\right)^{1 / 3}$ ("poor solvent" regime). For $\tau<\left(u f^{2}\right)^{1 / 3}$ ("close to theta solvent" regime), the chain behaves like a charged macromolecule in a $\theta$-solvent. These two regimes are shown on the vertical axis of figure 1 . The collapse of a neutral chain would occur at $\tau>N^{-1 / 2}$ whereas a much larger value of $\tau$ is required to cause the polyelectrolyte to collapse. In fact the criterion is $\tau>g_{0}^{-1 / 2}$, which is equivalent to saying that each blob has to contain enough monomers to collapse independently. We now consider how the presence of some amount of salt in the solution modifies the chain conformation in these two regimes.

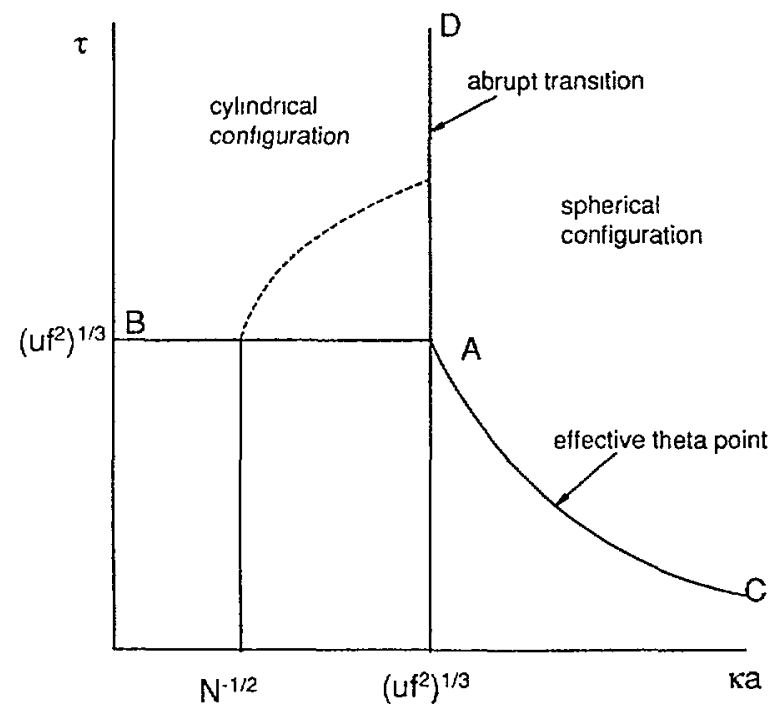

Fig. 1. - Conformations of a polyelectrolyte chain in a poor solvent as a function of solvent quality $\tau$ and inverse screening length $\kappa$ at fixed degree of ionization $f$.

\section{Polyelectrolyte in the "close to theta solvent" regime.}

We now consider the chain conformation in the presence of salt, assuming $0<\tau<\left(u f^{2}\right)^{1 / 3}$. For small salt concentrations we expect the salt to affect the flexibility of the chain of blobs rather than changing the behaviour on the level of one blob. More precisely, we expect the blob characteristics (Eq. (1)) to remain independent of the salt concentration as long as $\kappa^{-1}>D_{0}$. However, the salt affects the long range behaviour of the chain by introducing a finite persistence length $L_{\mathrm{p}}$ for the chain of blobs. For $\tau=0$ this has been calculated by KK [6] by modifying the original theory of Odijk and Houwaart [12] for strongly charged polyelectrolytes. The argument originally given for 
the line of monomers in the strongly charged case was applied directly to the line of blobs in the weakly charged case, and gives

$$
L_{\mathrm{p}} \sim \frac{\ell f^{2} g_{0}^{2}}{\kappa^{2} D_{0}^{2}} \sim \frac{\left(u f^{2}\right)^{1 / 3}}{\kappa^{2} a}
$$

We know that in the absence of salt : (i) the chain is unaffected by the short range interactions as long as $\tau<\left(u f^{2}\right)^{1 / 3}$; (ii) the collapse of the chain at $\tau \sim\left(u f^{2}\right)^{1 / 3}$ occurs in each blob separately. Since the size of the blob is unaffected by the salt as long as $\kappa D_{0}<1$, we expect the collapse transition to occur at the same value of $\tau$ (the line $A B$ of the figure), independently of the salt concentration. In particular $L_{\mathrm{p}}$ should still be given by equation (6) in the regime $0<\tau<\left(u f^{2}\right)^{1 / 3}$, in spite of the fact that $\tau$ is now non-zero.

As long as $\kappa a<N^{-1 / 2}, L_{\mathrm{p}}>L$ and the chain conformation is not modified by the presence of salt. For $\kappa a>N^{-1 / 2}$ the chain becomes flexible with a persistence length $L_{\mathrm{p}}$ (see the horizontal axis of Fig. 1). When $\kappa a \sim\left(u f^{2}\right)^{1 / 3}$ both the persistence length and the screening length shrink to the blob size $D_{0}$ and the notion of the electrostatic blob looses its meaning. If the concentration of added salt further increases, the theory of KK (which is for $\tau=0$ ) predicts that the chain behaves like a swollen chain with an electrostatic excluded volume $v_{\mathrm{el}}=4 \pi \ell f^{2} \kappa^{-2}$. This is because the screened Coulomb interation is short range and may be replaced by an excluded volume pseudopotential $v_{\mathrm{el}} \delta(r)$. In our case for $\tau \neq 0$, when $\kappa D_{0}>1$, the attractive short-range interactions become progressively more important and the chain is governed by the effective excluded volume parameter :

$$
v_{\text {eff }} \sim-\tau a^{3}+4 \pi \ell f^{2} \kappa^{-2}
$$

This is initially positive, but becomes negative for $\kappa^{2} a^{2}>u f^{2} / \tau$ (the line AC in the diagram). The chain therefore changes smoothly from swollen to collapsed on the addition of salt, passing through an effective $\theta$-point at the line $A C$.

This may be compared to the similar behaviour which is predicted for polyampholytes in reference [13]. These are polymers containing both positive and negative charges randomly distributed on the same chain. The net electrostatic interactions are attractive in this case. An effective $\theta$ point exists in a good solvent, where the repulsive excluded volume interactions balance the attractive electrostatic interactions.

We note, in addition, that $\mathrm{KK}$ divide the intermediate region of the horizontal axis $\left(N^{-1 / 2}<\right.$ $\kappa a<\left(u f^{2}\right)^{1 / 3}$ ) into two, according to the value of the excluded volume parameter between the different sections of length $L_{\mathrm{p}}$ within the chain of blobs. The same distinction can be applied here but we have not included it in the figure for sake of simplicity.

\section{Polyelectrolyte in the "poor solvent" regime.}

For $\tau>\left(u f^{2}\right)^{1 / 3}$ and in absence of salt, the chain is a line of collapsed blobs (Sect. 1). As in the previous section, for small salt concentrations we expect the salt to affect the flexibility of the chain of blobs rather than changing the behaviour on the level of one blob. We predict the persistence length for the poor solvent case following exactly the argument of $\mathrm{KK}[6]$ :

$$
L_{\mathrm{p}} \sim \frac{\ell f^{2} g^{2}}{\kappa^{2} D_{0}^{2}} \sim \frac{\tau^{2}}{\kappa^{2} a\left(u f^{2}\right)^{1 / 3}}
$$

This becomes equal to $L$ when $\kappa^{2} a^{2} \sim \tau^{3} /\left(N u f^{2}\right)$, shown as a dotted line in figure 1. $L_{\mathrm{p}}$ shrinks on increasing the salt concentration, but at the point $\kappa D_{0} \sim 1$ the persistence length is still much 
larger than $D_{0}$. In fact on the upper part of the line $\mathrm{AD}$ the chain is still one single rod at this point $\left(L_{\mathbf{p}}>L\right)$. On the other hand, once $\kappa D_{0}>1$ the repulsion between neighbouring blobs is screened, and the argument leading to the blob size $g$ (Eq. (3)) makes no further sense. Moreover the effective excluded volume (Eq. (6)) is negative at this point. This suggests that there is a discontinuous change at $\kappa D_{0} \sim 1$ from a cylindrical configuration (or a chain of blobs with large persistence length) to a spherical collapsed configuration with excluded volume $v_{\text {eff }}$. We give a more detailed argument to justify this below. There is again a parallel with the polyampholyte chain in a good solvent [13] where the collapse is smooth (passing through an effective $\theta$-point) at small excluded volume, and abrupt for large excluded volume.

For $\tau<\left(u f^{2}\right)^{1 / 3}$ (see Sect. 2) the monomer density inside the chain is that of a Gaussian chain, that is very low. It is clear that in this case the solvent and the salt can enter into the region occupied by the chain. For $\tau>\left(u f^{2}\right)^{1 / 3}$ the monomer density is that of a collapsed chain $\rho \sim \tau / a^{3}$. We are considering weakly charged chains - where the collapse of the polyelectrolyte occurs for $\tau \ll 1$ - and we will therefore assume that the density inside the chain is still rather low, and that the solvent and salt are still free to enter into the region occupied by the chain. This means that providing the dimensions of the chain are large compared to the screening length, the electrostatic interactions within the polymer are screened and behave like a contribution to the excluded volume.

We will now compare more carefully the energies of the cylinder and the sphere configurations. We will forget for the moment the finite persistence length, remembering that for at least part of the line $\mathrm{AD}$ the predicted transition is directly from cylinder to sphere. Suppose that $N \gg g$, so that the axial ratio $L / D$ of the cylinder is large, and we can forget the end effects. Omitting numerical constants, the free energy of the cylinder can be written

$$
\frac{F_{\text {cyl }}}{k T} \sim-N \tau^{2}+\tau^{2} \frac{L D}{a^{2}}+f^{2} \tau^{2} \frac{\ell L D^{4}}{a^{6}}
$$

The first term is the gain in energy of " $k T$ per thermal blob" when the chain collapses, which is the same as it would be for a neutral polymer. It is the real excluded volume $-\tau a^{3}$ which counts here, and not $v_{\text {eff }}$, since we assume the diameter is smaller than the screening length. The second term is the surface energy, which is significant due to the large surface area of the cynlinder. The charge density inside the cylinder is $f e \rho$, hence an electrostatic energy of order $(f e \rho)^{2} D^{4} L / \varepsilon$, which is the third term of equation (8). We have omitted the logarithmic factor in the third term $\left(\sim \ln (L / D)\right.$ in absence of salt, or $\ln \left(\kappa^{-1} / D\right)$ in presence of salt) since it will not greatly affect the scaling behaviour. This is equivalent to the argument of Khokhlov [5] that the electrostatic energy is dominated by the repulsion between nearest neighbour blobs of size $D$. Minimizing equation (8) with respect to $D$ at fixed volume $L D^{2} \sim N a^{3} \tau^{-1}$ gives $D=D_{0}$, and $L$ given by equation (4). The minimum energy of any cylindrical configuration is therefore

$$
\frac{F_{\text {cycl min }}}{k T} \sim-N \tau^{2}+N \tau\left(u f^{2}\right)^{1 / 3}
$$

We now consider a sphere of radius $R>\kappa^{-1}$. Screening is effective inside the sphere, so the modified excluded volume applies in this case (Eq. (6)). The sphere radius is therefore $R \sim$ $\left(N a^{3} / v_{\text {eff }}\right)^{1 / 3} a$. The energy gained on collapse is

$$
\frac{F_{\mathrm{sph}}}{k T} \sim-N\left(\tau-\frac{u f^{2}}{\kappa^{2} a^{2}}\right)^{2} \sim-N \tau^{2}+2 N \tau \frac{u f^{2}}{\kappa^{2} a^{2}}-
$$


The third term in the expansion is negligible close to the line $\mathrm{AD}$, where $\tau \gg \frac{u f^{2}}{\kappa^{2} a^{2}}$. There is a surface energy for the sphere which we have not written in equation (10) since it is negligible compared to the other terms. However, due to the surface energy the sphere will always have a minimum in energy relative to small increases of the axial ratio $L / D$. If on the other hand the axial ratio is increased to such an extent that $\kappa D<1$, equation (8) is applicable for the energy, not equation (10). There is therefore a second minimum in free energy as a function of $L / D$ corresponding to the cylinder of equation (9) (providing $\kappa D_{0}<1$ ). Comparison of equations (9) and (10) shows that the cylinder is preferred if $N \tau\left(u f^{2}\right)^{1 / 3}<N \tau \frac{u f^{2}}{\kappa^{2} a^{2}}$ i.e. if $\kappa D_{0}<1$. In summary, if $\kappa^{-1}>N^{1 / 3} \tau^{-1 / 3}$ the interactions are unscreened even for the sphere. The cylinder is the only minimum energy configuration. For $D_{0}<\kappa^{-1}<N^{1 / 3} \tau^{-1 / 3}$ there are two minima, but the cylinder is again preferred. For $\kappa^{-1}<D_{0}$ the sphere is preferred. There is therefore an abrupt change in the preferred axial ratio at the point $\kappa D_{0} \sim 1$.

\section{Discussion and conclusions.}

In the preceeding section we have shown that there should be a rapid change in configuration over a narrow range of salt concentration, which cannot be described as a smooth crossover between scaling regimes. We wish to stress that this conformational change has nothing to do with counterion condensation, and comes only from the competition between the two types of interactions between the monomers. As such it should be easily visible in computer simulations, for which the treatment of monomer-monomer interactions is relatively easy, but the explicit inclusion of mobile counterions requires much more computational time. Simulations of a polyelectrolyte chain in a $\theta$ solvent (Christos and Carnie [15]) show clearly a change from Gaussian to extended configurations as the fraction of charged monomers is increased. This is seen by calculation of the aspect ratio and the relative distributions of the monomers in space. More recently [16] the simulations have been extended to include mobile counterions and byions. Distributions of the ions about the chain can be calculated but the chains appear to be too short to test scaling theories. Hooper et al. [17] have simulated a polyelectrolyte in a poor solvent at various salt concentrations. The results seem in reasonable agreement with the theories discussed here in absence of salt, although no quantitative interpretation of the data was given. No clear evidence was seen for the abrupt transition predicted here in the presence of salt. This may again be because the chains are relatively short.

We have been assuming up to now that $f$ is fixed and $\tau$ and $\kappa$ are variables. We could of course treat $\tau$ as fixed, and consider $f$ and $\kappa$ as variables. This is more appropriate if we wish to consider the annealed polyelectrolytes of reference [9]. This time the mean number of charges on a chain is fixed by the solution $\mathrm{pH}$, but the exact number of charges on any one chain may vary. The rate of change of electrical free energy of the chain with addition of charges $\mu_{\mathrm{el}}(f)=\frac{1 \partial F_{\mathrm{el}}}{N \partial f}$ was shown to be decreasing function of $f$ in the region where the chain is in the collapsed cylindrical configuration (free energy in Eq. (9)). This leads to the possibility of coexistence of two species of chain with different charge fractions $f$, and may explain the "kinks" observed in certain titration curves (see experimental references in [9]). In absence of salt $\mu_{\mathrm{el}}$ is a decreasing function of $f$ in the range $\tau / N<u f^{2}<\tau^{3}$. In the presence of salt the same argument may be repeated, and we summarize the result here. For $\kappa^{-1}>N^{1 / 3} \tau^{-1 / 3}$ the behaviour will be as in absence of salt. If $a \tau^{-1}<n^{-1}<N^{1 / 3} \tau^{-1 / 3}$ the range of $f$ in which the cylindrical configuration exists is reduced, due to the abrupt collapse predicted above. Therefore $\mu_{\mathrm{el}}$ decreases with $f$ only in the range $(\kappa a)^{3}<u f^{2}<\tau^{3}$. When $\kappa a>\tau$ this range shrinks to zero because the collapsed 
cylindrical configuration no longer exists for any value of $f$. Strictly speaking the presence of the region of $\mu_{\mathrm{el}}$ decreasing with $f$ is not sufficient to cause the instability, since it may be dominated by entropic factors. These entropic factors can be calculated exactly as in reference [9]. In any case the coexistence of the two chain species and the anomalies in the titration curves should disappear in the presence of sufficient salt.

In conclusion, we have shown that the competition between short range attractive interactions and long range repulsive Coulomb interactions for a polyelectrolyte in a poor solvent may lead to a variety of different conformations of the chain. The chain configuration is affected strongly by the addition of salt. We have neglected the counterions, since for weakly charged chains in the dilute solution limit which we consider here, there is a negligible number of counterions in the region occupied by a chain. The configuration of one isolated polyelectrolyte chain is seen to be a non-trivial theoretical problem, and we hope to see verification of these theories from experiment and computer simulation.

\section{Acknowledgements.}

We thank Prof. J. F. Joanny for much useful help and advice. Paul Higgs was supported by the Commission of the European Communities under contract SC1000263.

\section{References}

[1] De Gennes P. G., Pincus P., Velasco R. M., Brochard F., J. Phys. France 37 (1976) 1461.

[2] PFEUTY P., J. Phys. Colloq. France 39 (1978) C2-149.

[3] ODIJK T., Macromolecules 12 (1979) 686.

[4] Hayter J., JanNinK G., BRochaRd-Wyard F., DE Gennes P. G., J. Phys. Lett. France 41 (1980) L451.

[5] KHOKHLOV A. R., J. Phys. A 13 (1980) 979.

[6] KhoKhKlov A. R., KhaChaturian K. A., Polymer 23 (1982).

[7] PINCUS P., WITTEN T., Europhys. Lett. 3 (1987) 315; Cohen J., Priel Z, Rabin Y., J. Chem. Phys. 88 (1988) 7111.

[8] BORUE V., ERUKHIMOVICH I., Macromolecules 21 (1988) 3240.

[9] JOANny J. F, LEIBLER L., J. Phys. France 51 (1990) 547.

[10] Manning G., J. Chem Phys. 51 (1969) 924.

[11] RAPHAEl E., JOANNY J. F., Europhys. Lett. (submitted 1990).

[12] Odijk T., Houwaart A. C., J. Polym. Sci. Polym. Phys. Ed. 16 (1978) 627.

[13] Higgs P. G., JoAnny J. F., J. Chem. Phys. (submitted 1990).

[14] DE GENNEs P. G., Scaling concepts in Polymer Physics (Cornell Univer. Press, Ithaca) 1985.

[15] Christos G. A., Carnie S. L., J. Chem. Phys. 91 (1989) 439.

[16] Christos G. A., CARniE S. L., J. Chem. Phys. 92 (1990) 7661.

[17] Hooper H. H., Beltran S., Sassi A. P., Blanch H. W., Prausnitz J. M., J. Chem. Phys. 93 (1990) 2715.

Cet article a été imprimé avec le Macro Package "Editions de Physique Avril 1990". 